\title{
Domino tilings for augmented Aztec rectangles and their chains
}

\author{
Hyunggi Kim \\ Department of Mathematical Science \\ Seoul National University \\ Seoul 08826, Korea \\ hyunggi@snu.ac.kr
}

\author{
Sangyop Lee* \\ Department of Mathematics \\ Chung-Ang University \\ Seoul 06974, Korea \\ sylee@cau.ac.kr
}

\author{
Seungsang $\mathrm{Oh}^{\dagger}$ \\ Department of Mathematics \\ Korea University \\ Seoul 02841, Korea \\ seungsang@korea.ac.kr
}

Submitted: Nov 28, 2018; Accepted: Jun 20, 2019; Published: Jul 5, 2019

(C) The authors. Released under the CC BY-ND license (International 4.0).

\begin{abstract}
Much work has been done to count the number of domino tilings for Aztec diamonds and augmented Aztec diamonds. Augmented Aztec rectangles and their chains are generalizations of these shapes. In this paper, we use Delannoy paths to count the number of domino tilings for these rectangles and their chains.
\end{abstract}

Mathematics Subject Classifications: 05A15, 05B45, 05C70

\section{Introduction}

The Aztec diamond of order $n$ is the union of unit squares with integral corners $(x, y)$ satisfying $|x|+|y| \leqslant n+1$ in the Cartesian coordinate system of $\mathbb{R}^{2}$. A domino is a 1-by-2 or 2-by-1 rectangle. A domino tiling of a region is a set of non-overlapping dominoes covering the region. Examples are shown in Figure 1.

The enumeration of domino tilings for the Aztec diamond is a problem of rich content in both enumerative combinatorics and statistical mechanics. This object appears first

\footnotetext{
* Supported by the National Research Foundation of Korea Grant funded by the Korean Government (NRF-2016R1D1A1B03935326).

${ }^{\dagger}$ The corresponding author was supported by the National Research Foundation of Korea(NRF) grant funded by the Korea government(MSIP) (No. NRF-2017R1A2B2007216).
} 

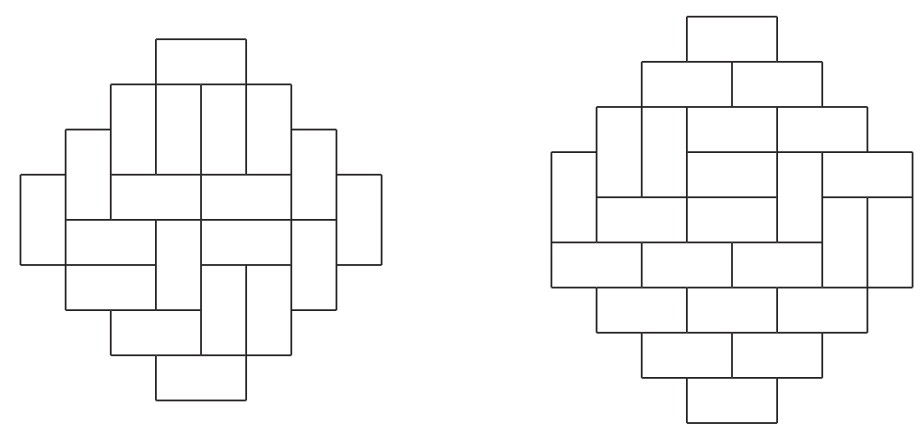

Figure 1: Domino tilings for the Aztec diamond and the augmented Aztec diamond of order 4

in [8] to present exact results for the asymptotic molecular freedom of square lattices of non-standard boundary shapes. Indeed, the number of domino tilings for a region is very sensitive to boundary conditions [11, 12]. Furthermore, from the viewpoint of statistical mechanics, tilings for large Aztec diamonds exhibit a striking feature: the Arctic circle theorem proved by Jockusch, Propp and Shor [9] says that a random domino tiling for a large Aztec diamond tends to be frozen outside a certain circle.

The Aztec diamond theorem, proved first by Elkies, Kuperberg, Larsen and Propp [4], states that the number of domino tilings for the Aztec diamond of order $n$ is equal to $2^{n(n+1) / 2}$. Later, simpler proofs are given in $[1,2,3,5]$. More interesting patterns related to the Aztec diamond with some squares removed have been studied and Propp gave a survey of these works [13].

The augmented Aztec diamond is obtained from the Aztec diamond by replacing the two long rows in the middle with three rows. See Figure 1. The number of domino tilings for the augmented Aztec diamond of order $n$ is shown by Sachs and Zernitz [14] to be $\sum_{k=0}^{n}\left(\begin{array}{l}n \\ k\end{array}\right) \cdot\left(\begin{array}{c}n+k \\ k\end{array}\right)$, which is known as the Delannoy numbers.

In this paper, we consider three variants of the augmented Aztec diamond, which are augmented Aztec rectangles and their vertical and horizontal chains shown in Figure 2. We count the number of domino tilings for each of these variants.

The augmented Aztec rectangle, which is a slanted rectangular variation of the augmented Aztec diamond, will be treated in Section 2. We show that the number of domino tilings for the augmented Aztec rectangle is the two-variable Delannoy number by constructing a bijection between domino tilings and Delannoy paths.

In Section 3, a chain of augmented Aztec rectangles is defined as the union of augmented Aztec rectangles overlapping vertically or horizontally, and we count the number of domino tilings for this shape.

\section{Augmented Aztec rectangle}

Let $p$ and $q$ be positive integers. An augmented Aztec rectangle of size $(p, q)$, denoted $\mathcal{R}_{(p, q)}$, is a natural generalization of the augmented Aztec diamond, which is the region having $p$ and $q$ diagonally consecutive squares on its southwest and northwest sides, re- 

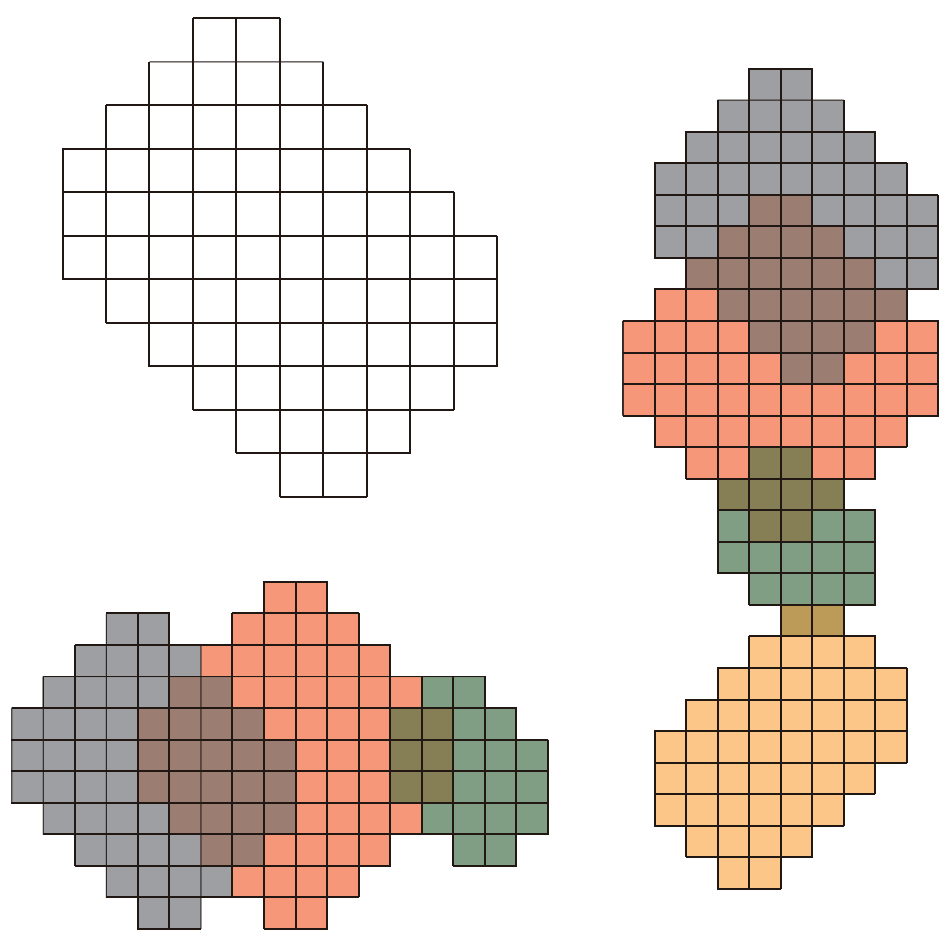

Figure 2: Three variants of the augmented Aztec diamond

spectively. See Figure 3 for $\mathcal{R}_{(6,4)}$. We mention that $\mathcal{R}_{(p, q)}$ has three squares on both leftmost and rightmost columns and two squares on both top and bottom rows. In particular, $\mathcal{R}_{(p, p)}$ is an augmented Aztec diamond of order $p$. Note that $\mathcal{R}_{(p, q)}$ is the union of $2(p q+p+q)$ unit squares.

We now color the squares of $\mathcal{R}_{(p, q)}$ black and white so that any two adjacent squares have opposite colors as the checkerboard and that the middle square of the leftmost column is colored black. This middle black square is called a pivot (black) square. Note that the middle square of the rightmost column is colored white. The 'imaginary' black square which is not contained in $\mathcal{R}_{(p, q)}$ but adjacent to this white square is called a ghost (black) square. See Figure 4.

Now we rotate $\mathcal{R}_{(p, q)}$ counter-clockwise by $45^{\circ}$, shrink in uniform scaling by $\frac{1}{\sqrt{2}}$, and place the center of the pivot black square at $(0,0)$ in the Cartesian coordinate so that the center of each black square has non-negative integer coordinates. From now on, by $\mathcal{R}_{(p, q)}$, we mean this rotated augmented Aztec rectangle.

We define the dual lattice graph of $\mathcal{R}_{(p, q)}$, denoted $\Gamma_{(p, q)}$, to be the graph whose vertices correspond to the center points of black squares and an extra point $(p, q)$, and two vertices are connected by an edge whenever the corresponding points are at distance 1 or a pair $\{(i, j),(i+1, j+1)\}$, as drawn in the upper picture in Figure 4 . The center points $(0,0)$ and $(p, q)$ of the pivot and ghost black squares are called pivot and ghost vertices, respectively.

To tile $\mathcal{R}_{(p, q)}$, we need $p q+p+q$ dominoes of four types as the lower pictures in Figure 4. A domino is called an sw-, nw-, se- or ne-domino, depending on whether the 


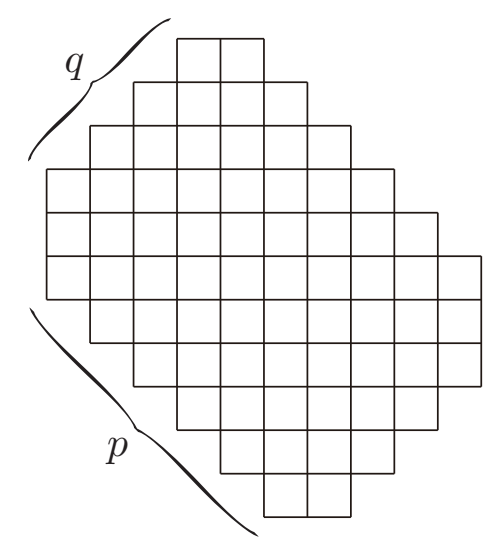

Figure 3: $\mathcal{R}_{(6,4)}$
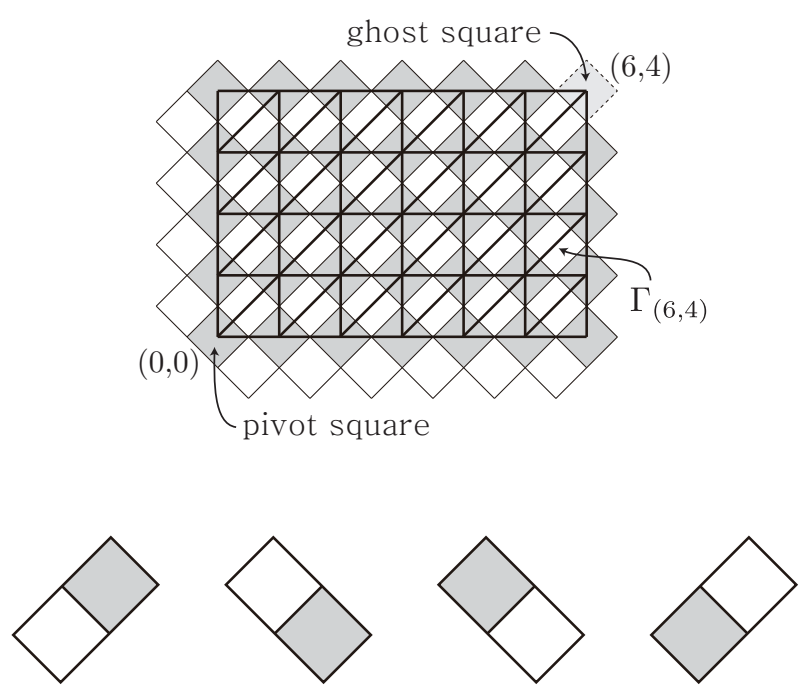

Figure 4: $\Gamma_{(6,4)}$ and four dominoes

white square is located in the southwest, northwest, southeast or northeast of the black square. For two adjacent squares $A$ and $B$, if $A$ is located in the southwest of $B$, then $A$ is said to be sw-adjacent to $B$ and $B$ is said to be ne-adjacent to $A$.

A Delannoy path in $\Gamma_{(p, q)}$ is a lattice path with steps in $\{(0,1),(1,0),(1,1)\}$ from $(0,0)$ to $(p, q)$ as drawn in Figure 5 . The number of Delannoy paths in $\Gamma_{(p, q)}$ is known as Delannoy number $D(p, q)$, which is given by

$$
D(p, q)=\sum_{k=0}^{\min \{p, q\}}\left(\begin{array}{c}
p+q-k \\
p
\end{array}\right)\left(\begin{array}{l}
p \\
k
\end{array}\right) .
$$

We define a map $\Phi$ from the set of domino tilings for $\mathcal{R}_{(p, q)}$ to the set of subgraphs of $\Gamma_{(p, q)}$ by using the following: 


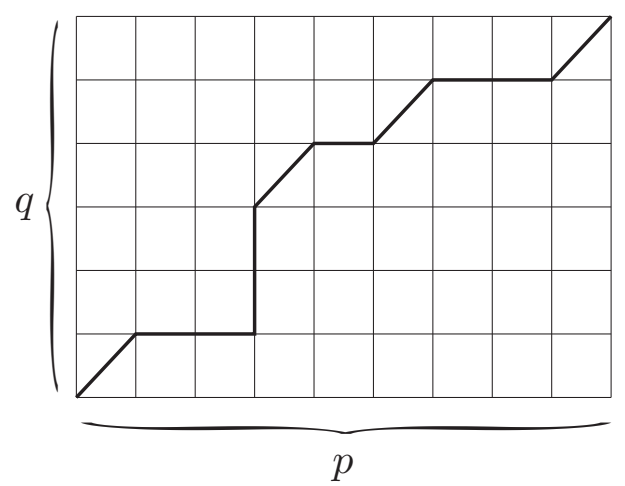

Figure 5: A Delannoy path

Delannoy Path Replacement. We replace each nw-, se- or ne-domino of a domino tiling for $\mathcal{R}_{(p, q)}$ by an edge of $\Gamma_{(p, q)}$ corresponding to the step $(0,1),(1,0)$ or $(1,1)$, respectively, and starting at the center of the black square of the domino as drawn in Figure 6, while we ignore sw-dominoes.
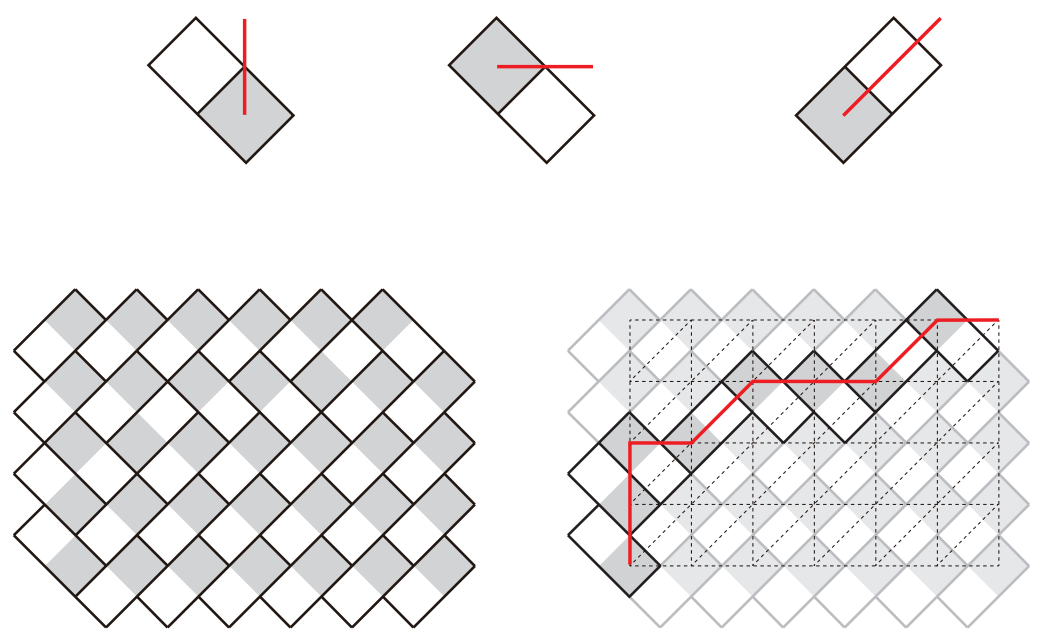

Figure 6: Delannoy path replacement of dominoes

The map $\Phi$ is called the Delannoy path map and nw-, se- and ne-dominoes are called $D$-dominoes. The following theorem provides a useful property of the Delannoy path map; its image of a domino tiling for $\mathcal{R}_{(p, q)}$ is a Delannoy path running from $(0,0)$ to $(p, q)$. So we restrict the codomain of the Delannoy path map to the set of Delannoy paths from $(0,0)$ to $(p, q)$. Let $\rho_{(p, q)}$ denote the number of domino tilings for $\mathcal{R}_{(p, q)}$.

Theorem 1. Delannoy path map $\Phi$ is a bijection between domino tilings for an augmented Aztec rectangle $\mathcal{R}_{(p, q)}$ and Delannoy paths from $(0,0)$ to $(p, q)$. Therefore the number of 
domino tilings for $\mathcal{R}_{(p, q)}$ is given by

$$
\rho_{(p, q)}=\sum_{k=0}^{\min \{p, q\}}\left(\begin{array}{c}
p+q-k \\
p
\end{array}\right)\left(\begin{array}{l}
p \\
k
\end{array}\right) .
$$

Proof. The following two claims show that the image of $\Phi$ when applied to a domino tiling $T$ for $\mathcal{R}_{(p, q)}$ is exactly a Delannoy path from $(0,0)$ to $(p, q)$.

Claim 2. $\Phi(T)$ contains a Delannoy path from the pivot vertex $(0,0)$ to the ghost vertex $(p, q)$.

Proof. In $T$, the pivot vertex $(0,0)$ must be covered by a D-domino, say $D_{1}$, since the pivot black square cannot be covered by an sw-domino. By the Delannoy path replacement of $D_{1}$, we get a single-edge path $P_{1}$ in $\Gamma_{(p, q)}$, starting at $\left(x_{0}, y_{0}\right)=(0,0)$ and ending at the vertex $\left(x_{1}, y_{1}\right)$, where $\left(x_{1}, y_{1}\right)$ is $(0,1),(1,0)$ or $(1,1)$ according to whether $D_{1}$ is an nw-domino, se-domino or ne-domino.

Next, we consider the black square whose center is $\left(x_{1}, y_{1}\right)$. It is indeed ne-adjacent to the white square of $D_{1}$. Then this black square must be covered by another D-domino, say $D_{2}$, in $T$. We extend $P_{1}$ to a new path $P_{2}$ by adjoining the edge obtained by the Delannoy path replacement of $D_{2}$ as before. Note that the terminal point $\left(x_{2}, y_{2}\right)$ of $P_{2}$ is given by

$$
\left(x_{2}, y_{2}\right)= \begin{cases}\left(x_{1}, y_{1}+1\right) & \text { if } D_{2} \text { is an nw-domino } \\ \left(x_{1}+1, y_{1}\right) & \text { if } D_{2} \text { is an se-domino } \\ \left(x_{1}+1, y_{1}+1\right) & \text { if } D_{2} \text { is an ne-domino }\end{cases}
$$

We continue this process to construct a sequence of paths $P_{1}, P_{2}, \ldots$ so that $P_{k+1}$ is obtained from $P_{k}$ by adjoining a single edge of $\Gamma_{(p, q)}$ corresponding to the D-domino $D_{k+1}$ which covers the terminal point $\left(x_{k}, y_{k}\right)$ of $P_{k}$. The terminal point $\left(x_{k+1}, y_{k+1}\right)$ of the new path $P_{k+1}$ is determined by $\left(x_{k}, y_{k}\right)$ and $D_{k+1}$ in a similar way as (2.1). In particular, if this black square is located at the boundary on the right/top side of $\mathcal{R}_{(p, q)}$, then $D_{k+1}$ is always an nw-/se-domino and so $P_{k+1}$ is obtained from $P_{k}$ by adjoining a $(0,1) /(1,0)$ step.

It is clear that the above process stops in finite steps since the region $\mathcal{R}_{(p, q)}$ is bounded. More precisely, we cannot continue this process when the terminal point $\left(x_{k}, y_{k}\right)$ does not belong to any black square in $\mathcal{R}_{(p, q)}$. In $\mathcal{R}_{(p, q)}$, every white square $S_{w}$ has a black square which is ne-adjacent to $S_{w}$, as long as the center of $S_{w}$ is not $\left(p-\frac{1}{2}, q-\frac{1}{2}\right)$. Therefore this process stops when $P_{k}$ reaches the ghost vertex $(p, q)$.

Let $P$ be the Delannoy path constructed in Claim 2. Assume that there is a D-domino $D^{\prime}$ away from $P$. By using the above process, we can construct another path $P^{\prime}$ starting at the center of the black square of $D^{\prime}$.

Claim 3. The above two paths $P$ and $P^{\prime}$ in $\Phi(T)$ do not intersect. 
Proof. Assume that $P$ and $P^{\prime}$ intersect. Let $(a, b)$ be the first vertex at which they meet (clearly $(a, b) \neq(0,0))$. Let $S_{b}$ be the black square centered at $(a, b)$ (or the ghost black square if $(a, b)=(p, q))$ and $S_{w}$ be the white square centered at $\left(a-\frac{1}{2}, b-\frac{1}{2}\right)$. Since $S_{b}$ is either covered by a D-domino or the ghost black square, $S_{w}$ cannot be covered by an sw-domino. Therefore $S_{w}$ must be covered by a D-domino $D$ in $T$, which is adjacent to $S_{b}$. The center $\left(a^{\prime}, b^{\prime}\right)$ of the black square of $D$ must be uniquely determined to be one of $(a-1, b),(a, b-1)$ or $(a-1, b-1)$. This contradicts the choice of $(a, b)$ because both $P$ and $P^{\prime}$ pass through $\left(a^{\prime}, b^{\prime}\right)$.

The proof of Claim 2 shows that $P^{\prime}$ must terminate at $(p, q)$, contradicting Claim 3. Therefore the dominoes in $T$ away from $P$ are all sw-dominoes. As desired, this implies that $\Phi(T)$ itself is the Delannoy path $P$.

It remains to show that the map $\Phi$ is bijective. The injectivity follows immediately from the recipe of the Delannoy path replacement. We next show that $\Phi$ is surjective. We will construct a domino tiling of $\mathcal{R}_{(p, q)}$ from a given Delannoy path $P$ in $\Gamma_{(p, q)}$ by reversing the Delannoy path replacement. Suppose that $P$ consists of $m$ steps $s_{1}, \ldots, s_{m}$ in order. Replace each step $s_{i}$ by a D-domino $D_{i}$ in such a way that the center of its black square is placed at the starting point of $s_{i}$ and that

$$
D_{i}= \begin{cases}\text { an nw-domino } & \text { if } s_{i}=(0,1), \\ \text { an se-domino } & \text { if } s_{i}=(1,0), \\ \text { an ne-domino } & \text { if } s_{i}=(1,1) .\end{cases}
$$

Notice that the black square of $D_{i}$ has its center at the starting point of $s_{i}$ and the white square of $D_{i}$ is next to the ending point of $s_{i}$ in the southwest direction (see Figure 6 ). Two distinct D-dominoes $D_{i}$ and $D_{j}$ cannot share a square since the starting (resp. ending) points of $s_{i}$ and $s_{j}$ are different. Therefore the D-dominoes $D_{1}, \ldots, D_{m}$ do not overlap. Let $\mathcal{R}_{P}$ be the subregion of $\mathcal{R}_{(p, q)}$ consisting of these D-dominoes.

Let $\mathcal{R}_{P}^{c}$ be the complementary region of $\mathcal{R}_{P}$. It is enough to show that $\mathcal{R}_{P}^{c}$ can always be tiled only by sw-dominoes. Take any white square $S_{w}$ in $\mathcal{R}_{P}^{c}$ (so not centered at $\left(p-\frac{1}{2}, q-\frac{1}{2}\right)$ ). Let $S_{b}$ be the black square which is ne-adjacent to $S_{w}$. If $S_{b}$ is contained in $\mathcal{R}_{P}$, then the center of $S_{b}$ is the ending point of some step $s_{i}$ in $P$. From the construction of $\mathcal{R}_{P}, S_{w}$ is the white square of the D-domino $D_{i}$ corresponding to $s_{i}$ and so it must be contained in $\mathcal{R}_{P}$, a contradiction. Therefore both squares $S_{w}$ and $S_{b}$ are contained in $\mathcal{R}_{P}^{c}$, and we can cover them by an sw-domino. This implies that $\mathcal{R}_{P}^{c}$ can be tiled only by sw-dominoes since it has the same number of white squares and black squares. The proof of [1, Proposition 6.1] provides a similar proof of the bijectivity in a slight different point of view.

\section{Chains of augmented Aztec rectangles}

In this section, we consider a chain of augmented Aztec rectangles, a region $\mathcal{R}_{1} \cup \mathcal{R}_{2} \cup$ $\cdots \cup \mathcal{R}_{n}$ obtained by arranging finitely many augmented Aztec rectangles in such a way 
that $\mathcal{R}_{k}$ and $\mathcal{R}_{k+1}$ overlap for each $k$. See Figure 2. As in Section 2, we view these rectangles after rotating them counter-clockwise by $45^{\circ}$ and shrinking in uniform scaling by $\frac{1}{\sqrt{2}}$. Here, $\mathcal{R}_{k}=\mathcal{R}_{\left(p_{k}, q_{k}\right)}\left(a_{k}, b_{k}\right)$ is an augmented Aztec rectangle $\mathcal{R}_{\left(p_{k}, q_{k}\right)}$, the center of whose pivot black square is placed at $\left(a_{k}, b_{k}\right)$ with integer coordinates.

We say that two consecutive rectangles $\mathcal{R}_{k}$ and $\mathcal{R}_{k+1}$ overlap vertically if

$$
\left\{\begin{array}{l}
a_{k}<a_{k+1} \leqslant a_{k}+p_{k}<a_{k+1}+p_{k+1}, \\
b_{k+1}<b_{k} \leqslant b_{k+1}+q_{k+1}<b_{k}+q_{k},
\end{array}\right.
$$

and they overlap horizontally if

$$
\left\{\begin{array}{l}
a_{k}<a_{k+1} \leqslant a_{k}+p_{k}<a_{k+1}+p_{k+1}, \\
b_{k}<b_{k+1} \leqslant b_{k}+q_{k}<b_{k+1}+q_{k+1} .
\end{array}\right.
$$

In Figure 7, two rectangles overlap vertically in the left figure and overlap horizontally in the right figure, (Clockwise rotation by $45^{\circ}$ recovers their original positions, which are vertical and horizontal). For each case, we give an example of domino tilings and its corresponding Delannoy path family.
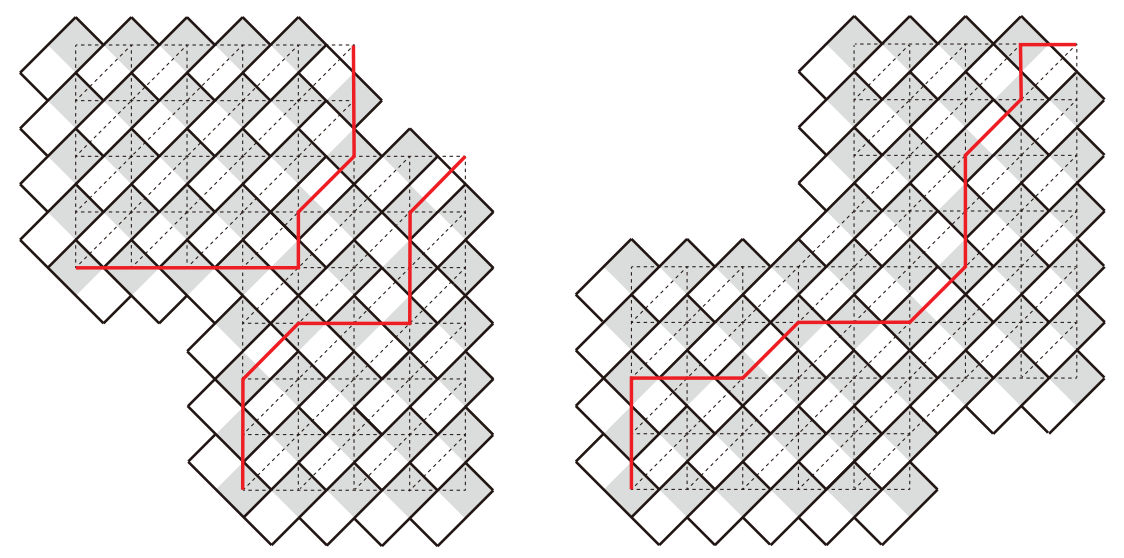

Figure 7: Overlapping augmented Aztec rectangles

Lemma 4. When two augmented Aztec rectangles overlap vertically or horizontally, their checkerboard colorings match in the overlapping region. Also, the overlapping region (and hence the total region) has the same number of black and white squares.

Proof. Suppose that two augmented Aztec rectangles

$$
\mathcal{R}_{\left(p_{k}, q_{k}\right)}\left(a_{k}, b_{k}\right) \quad \text { and } \quad \mathcal{R}_{\left(p_{k+1}, q_{k+1}\right)}\left(a_{k+1}, b_{k+1}\right)
$$

overlap vertically or horizontally. The center of each black square of the two rectangles has integer coordinates, while the center of each white square has half integer coordinates. This implies that their checkerboard colorings match in the overlapping region. 
One can easily count the number of the black and white squares in the overlapping region. Actually the overlapping region has the same number of black and white squares which is $\left(a_{k}+p_{k}-a_{k+1}+1\right)\left(b_{k+1}+q_{k+1}-b_{k}+1\right)$ if they overlap vertically, and is $\left(a_{k}+p_{k}-a_{k+1}+1\right)\left(b_{k}+q_{k}-b_{k+1}+1\right)-1$ if they overlap horizontally.

A union $\bigcup_{k=1}^{n} \mathcal{R}_{\left(p_{k}, q_{k}\right)}\left(a_{k}, b_{k}\right)$ is called a vertical chain of augmented Aztec rectangles if all pairs of consecutive augmented Aztec rectangles overlap vertically. Similarly we define a horizontal chain. Furthermore, a vertical chain is said to be simple if we have $a_{k+1}=a_{k}+p_{k}$ and $b_{k}=b_{k+1}+q_{k+1}$ in (3.1) for all $k$ so that two consecutive rectangles share exactly a pair of adjacent black and white squares. Similarly, a horizontal chain is said to be simple if we have $a_{k+1}=a_{k}+p_{k}$ and $b_{k+1}=b_{k}+q_{k}$ in (3.2) for all $k$ so that two consecutive rectangles are not laid overlapping each other but just adjacent. See Figure 8.
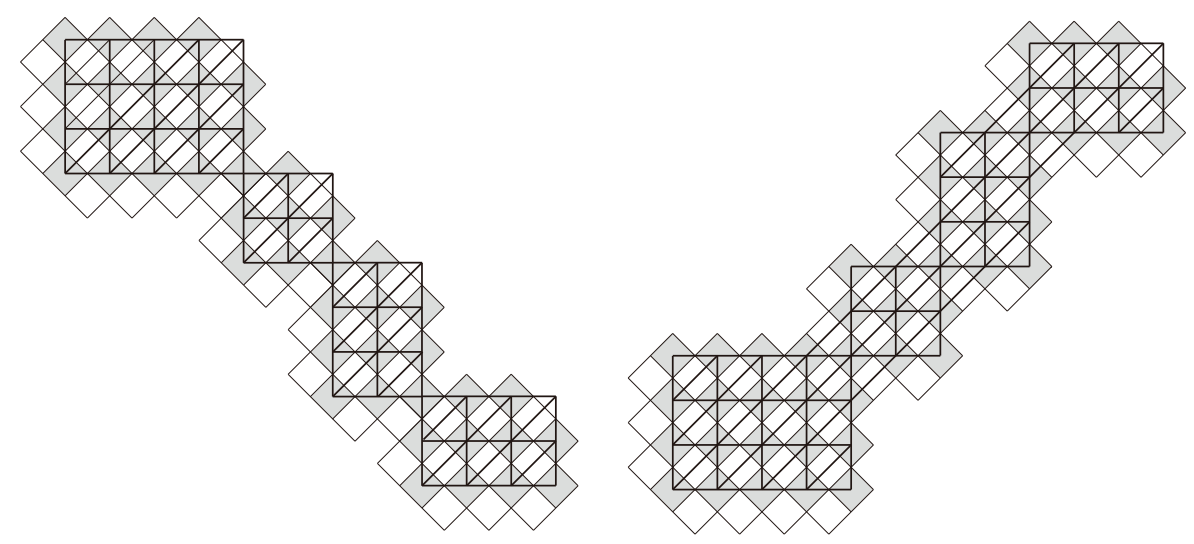

Figure 8: Two simple vertical and horizontal chains (clockwise rotation by $45^{\circ}$ recovers their original vertical and horizontal positions)

\subsection{Vertical chains of augmented Aztec rectangles}

The dual lattice graph $\Gamma_{v}$ of a vertical chain is defined as the union of the dual lattice graphs of all augmented Aztec rectangles, that is, $\Gamma_{v}=\bigcup_{k=1}^{n} \Gamma_{\left(p_{k}, q_{k}\right)}\left(a_{k}, b_{k}\right)$. It has $n$ pivot vertices $\left\{\left(a_{k}, b_{k}\right)\right\}$ and $n$ ghost vertices $\left\{\left(a_{k}+p_{k}, b_{k}+q_{k}\right)\right\}$. The vertical chain in Figure 2 and its dual lattice graph are drawn together in Figure 9.

Recall that $\rho_{(p, q)}$ is calculated in Theorem 1. Each $(i, j)$-entry $\rho_{\left(a_{j}+p_{j}-a_{i}, b_{j}+q_{j}-b_{i}\right)}$ of the matrix in the next theorem is the number of Delannoy paths from the $i$-th pivot vertex $\left(a_{i}, b_{i}\right)$ to the $j$-th ghost vertex $\left(a_{j}+p_{j}, b_{j}+q_{j}\right)$ in $\Gamma_{v}$. Here, we define

$$
\rho_{(p, q)}= \begin{cases}0 & \text { if } p<0 \text { or } q<0 \\ 1 & \text { if } \min \{p, q\}=0\end{cases}
$$

It is natural to define $\rho_{(p, 0)}=1$ because there is only one domino tiling for the configuration inside the dotted circle in Figure 9. 


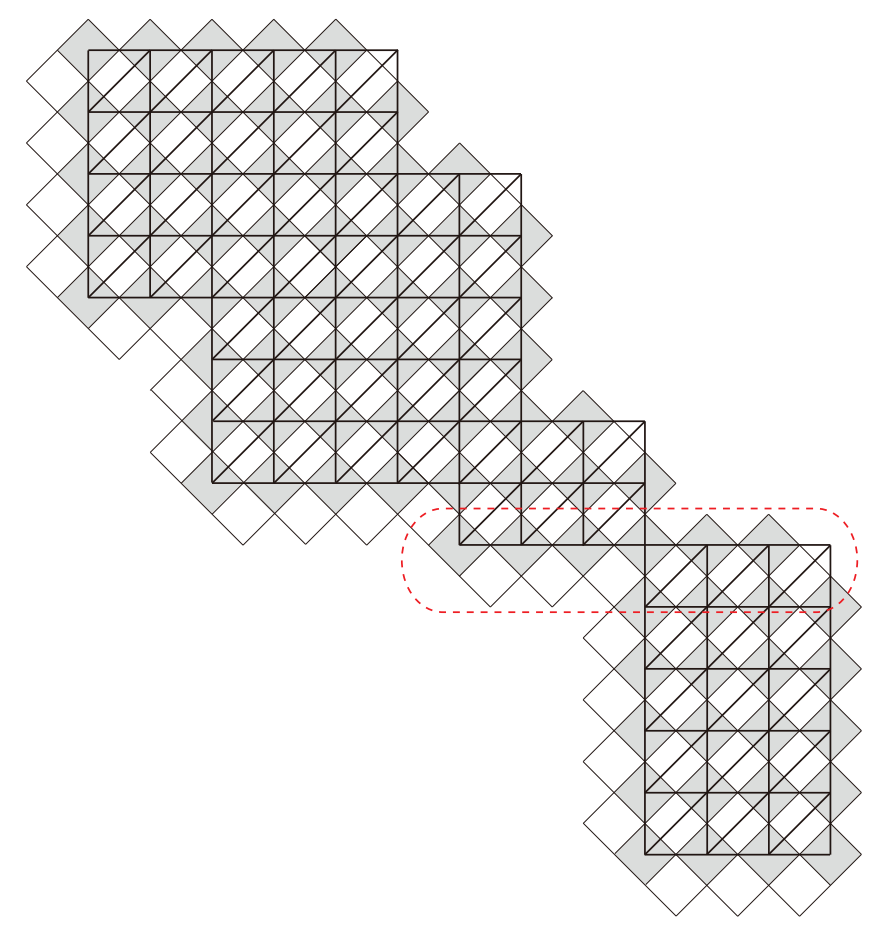

Figure 9: A vertical chain and its dual lattice graph $\Gamma_{v}$

Theorem 5. There is a bijection between domino tilings for a vertical chain

$$
\bigcup_{k=1}^{n} \mathcal{R}_{\left(p_{k}, q_{k}\right)}\left(a_{k}, b_{k}\right)
$$

and families of $n$ non-intersecting Delannoy paths, each running from $\left(a_{k}, b_{k}\right)$ to $\left(a_{k}+\right.$ $\left.p_{k}, b_{k}+q_{k}\right)$ in its dual lattice graph $\Gamma_{v}$. Furthermore, the number of domino tilings for this chain is given by the determinant of the $n \times n$ matrix

$$
\operatorname{det}\left[\rho_{\left(a_{j}+p_{j}-a_{i}, b_{j}+q_{j}-b_{i}\right)}\right]_{1 \leqslant i, j \leqslant n} .
$$

Proof. As before, we define a map $\Phi_{v}$ from the set of domino tilings for this vertical chain to the set of subgraphs of $\Gamma_{v}$ by using the Delannoy path replacement.

We first show that its image of a domino tiling $T$ is a family of $n$ non-intersecting Delannoy paths, each running from $\left(a_{k}, b_{k}\right)$ to $\left(a_{k}+p_{k}, b_{k}+q_{k}\right)$. We can apply the same path extension process starting from each pivot vertex $\left(a_{k}, b_{k}\right)$ as in the proof of Theorem 1 to get a uniquely defined Delannoy path $P_{k}$ running from $\left(a_{k}, b_{k}\right)$ to a ghost vertex $\left(a_{k^{\prime}}+p_{k^{\prime}}, b_{k^{\prime}}+q_{k^{\prime}}\right)$ for some $k^{\prime}=1,2, \ldots, n$ by Claim 2 . The indices $k$ and $k^{\prime}$ may be different because the path $P_{k}$ may leave the rectangle $\mathcal{R}_{\left(p_{k}, q_{k}\right)}\left(a_{k}, b_{k}\right)$ and go into another rectangle $\mathcal{R}_{\left(p_{k^{\prime}}, q_{k^{\prime}}\right)}\left(a_{k^{\prime}}, b_{k^{\prime}}\right)$.

By Claim 3, $\Phi_{v}(T)$ is a family of non-intersecting Delannoy paths $P_{1}, \ldots, P_{n}$, obviously each from $\left(a_{k}, b_{k}\right)$ to $\left(a_{k}+p_{k}, b_{k}+q_{k}\right)$ in $\Gamma_{v}$. Also, the dominoes in $T$ away from these paths are all sw-dominoes. 
The injectivity of $\Phi_{v}$ follows immediately from the Delannoy path replacement. A similar argument as in the proof of Theorem 1 establishes the surjectivity as follows. We construct a domino tiling of the chain from a given family of $n$ non-intersecting Delannoy paths $P_{1}, \ldots, P_{n}$ in $\Gamma_{v}$ by reversing the Delannoy path replacement. As before, for each $k$, the union of the D-dominoes which correspond to the steps of $P_{k}$ defines a subregion $\mathcal{R}_{P_{k}}$. Note that any two of these subregions do not overlap. Let $\mathcal{R}_{\cup P_{k}}^{c}$ be the complementary region of $\bigcup_{k=1}^{n} \mathcal{R}_{P_{k}}$. By Lemma 4 , the union $\bigcup_{k=1}^{n} \mathcal{R}_{\left(p_{k}, q_{k}\right)}\left(a_{k}, b_{k}\right)$ has the same number of black and white squares, and so does $\mathcal{R}_{\bigcup P_{k}}^{c}$. As before, for any white square in $\mathcal{R}_{\bigcup}^{c} P_{k}$, the black square which is ne-adjacent to the white square is also contained in $\mathcal{R}_{\cup}^{c} P_{k}$. This implies that $\mathcal{R}_{\bigcup P_{k}}^{c}$ can be tiled only by sw-dominoes, giving a domino tiling of $\bigcup_{k=1}^{n} \mathcal{R}_{\left(p_{k}, q_{k}\right)}\left(a_{k}, b_{k}\right)$ whose image under $\Phi_{v}$ is the family of $n$ non-intersecting Delannoy paths $P_{1}, \ldots, P_{n}$.

Applying Lindström-Gessel-Viennot lemma [6, 7, 10], the number of the families of these $n$ non-intersecting Delannoy paths is given by the determinant

$$
\operatorname{det}\left[\rho_{i j}\right]_{1 \leqslant i, j \leqslant n},
$$

where $\rho_{i j}$ denotes the number of Delannoy paths from $\left(a_{i}, b_{i}\right)$ to $\left(a_{j}+p_{j}, b_{j}+q_{j}\right)$. This number $\rho_{i j}$ is indeed $\rho_{\left(a_{j}+p_{j}-a_{i}, b_{j}+q_{j}-b_{i}\right)}$.

Corollary 6. The number of domino tilings for a simple vertical chain $\bigcup_{k=1}^{n} \mathcal{R}_{\left(p_{k}, q_{k}\right)}\left(a_{k}, b_{k}\right)$ is given by the determinant of the $n \times n$ matrix

$$
\operatorname{det}\left[\begin{array}{ccccc}
\rho_{\left(p_{1}, q_{1}\right)} & 1 & 0 & \ldots & 0 \\
1 & \rho_{\left(p_{2}, q_{2}\right)} & 1 & \ldots & 0 \\
0 & 1 & \rho_{\left(p_{3}, q_{3}\right)} & \ldots & 0 \\
\vdots & \vdots & \vdots & \ddots & \vdots \\
0 & 0 & 0 & 1 & \rho_{\left(p_{n}, q_{n}\right)}
\end{array}\right] .
$$

\subsection{Horizontal chains of augmented Aztec rectangles}

The dual lattice graph of a horizontal chain $\mathcal{R}_{1} \cup \mathcal{R}_{2} \cup \cdots \cup \mathcal{R}_{n}$ is defined as the union of the dual lattice graphs of all augmented Aztec rectangles with $2(n-1)$ extra edges,

$$
\Gamma_{h}=\bigcup_{k=1}^{n} \Gamma_{\left(p_{k}, q_{k}\right)}\left(a_{k}, b_{k}\right) \cup \bigcup_{k=1}^{n-1}\left\{e_{k}, e_{k}^{\prime}\right\},
$$

where each edge $e_{k}$ connects two vertices $\left(a_{k+1}-1, b_{k}+q_{k}\right)$ and $\left(a_{k+1}, b_{k}+q_{k}+1\right)$ and $e_{k}^{\prime}$ connects $\left(a_{k}+p_{k}, b_{k+1}-1\right)$ and $\left(a_{k}+p_{k}+1, b_{k+1}\right)$.

Unlike the vertical chain case, it has only one pivot vertex $\left(a_{1}, b_{1}\right)$ and only one ghost vertex $\left(a_{n}+p_{n}, b_{n}+q_{n}\right)$. Also, we need the extra edges $e_{k}$ (and $\left.e_{k}^{\prime}\right)$ because the black square centered at $\left(a_{k+1}-1, b_{k}+q_{k}\right)$ and the white square centered at $\left(a_{k+1}-\frac{1}{2}, b_{k}+q_{k}+\frac{1}{2}\right)$ are possibly covered by an ne-domino which is contained in neither $\mathcal{R}_{k}$ nor $\mathcal{R}_{k+1}$ but in 


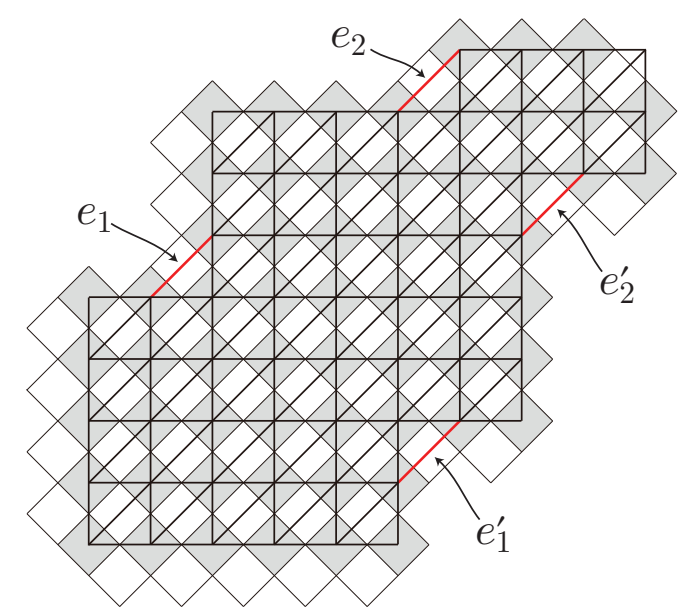

Figure 10: A horizontal chain and its dual lattice graph $\Gamma_{h}$

$\mathcal{R}_{k} \cup \mathcal{R}_{k+1}$. The edge $e_{k}$ is obtained from the Delannoy path replacement of this ne-domino. The horizontal chain in Figure 2 and its dual lattice graph are shown in Figure 10.

Consider a broken line segment $l_{k}(k=1,2, \ldots, n-1)$ which divides $\Gamma_{h}$ into two pieces as drawn in Figure 11. The lines $l_{k}$ serve as frontiers that need to be crossed, and every path is characterized first by the set of points where these frontiers are crossed, and then by the ways to interpolate paths between successive crossings, the latter being counted by Delannoy numbers. Each $l_{k}$, which starts and ends at the midpoints of the edges $e_{k}$ and $e_{k}^{\prime}$, is broken into a diagonal segment and (if necessary) a horizontal or vertical segment. More precisely, it starts with a diagonal segment proceeding until horizontal or vertical alignment with the end point is obtained, after which a horizontal or vertical segment toward that end point is added if needed. Each Delannoy path from $\left(a_{1}, b_{1}\right)$ to $\left(a_{n}+p_{n}, b_{n}+q_{n}\right)$ in $\Gamma_{h}$ always passes through $l_{k}$ exactly once. Let $M_{k}$ be the number of intersection points of $\Gamma_{h}$ with $l_{k}$, and $m_{k}$ the number of intersection points of $\Gamma_{h}$ with the diagonal part of $l_{k}$. Then

$$
\left\{\begin{array}{l}
M_{k}=2 \max \left\{a_{k}+p_{k}-a_{k+1}, b_{k}+q_{k}-b_{k+1}\right\}+3 \\
m_{k}=2 \min \left\{a_{k}+p_{k}-a_{k+1}, b_{k}+q_{k}-b_{k+1}\right\}+3
\end{array}\right.
$$

and we define $M_{0}=M_{n}=1$ for convenience.

We take two families $\left\{v(k, 1), \ldots, v\left(k, M_{k}\right)\right\}$ and $\left\{w(k, 1), \ldots, w\left(k, M_{k}\right)\right\}$, of vertices on either side of $l_{k}$ with the property that for each $i$ either two vertices $v(k, i)$ and $w(k, i)$ are the same point in $l_{k}$ or span an edge bisected by $l_{k}$. See Figure 11. More precisely, for $1 \leqslant i \leqslant m_{k}$, we have

$$
\left\{\begin{array}{l}
v(k, i)=\left(a_{k+1}+\left\lfloor\frac{i-2}{2}\right\rfloor, b_{k}+q_{k}-\left\lfloor\frac{i-1}{2}\right\rfloor\right) \\
w(k, i)=\left(a_{k+1}+\left\lfloor\frac{i-1}{2}\right\rfloor, b_{k}+q_{k}-\left\lfloor\frac{i-2}{2}\right\rfloor\right)
\end{array}\right.
$$


and for $m_{k}<i \leqslant M_{k}$, we have either

$$
\left\{\begin{array}{l}
v(k, i)=\left(a_{k+1}+\left\lfloor\frac{i-2}{2}\right\rfloor, b_{k+1}-1\right), \\
w(k, i)=\left(a_{k+1}+\left\lfloor\frac{i-1}{2}\right\rfloor, b_{k+1}\right)
\end{array}\right.
$$

if $a_{k}+p_{k}-a_{k+1} \geqslant b_{k}+q_{k}-b_{k+1}$ or

$$
\left\{\begin{array}{l}
v(k, i)=\left(a_{k}+p_{k}, b_{k}+q_{k}-\left\lfloor\frac{i-1}{2}\right\rfloor\right), \\
w(k, i)=\left(a_{k}+p_{k}+1, b_{k}+q_{k}-\left\lfloor\frac{i-2}{2}\right\rfloor\right)
\end{array}\right.
$$

if $a_{k}+p_{k}-a_{k+1}<b_{k}+q_{k}-b_{k+1}$ (with the figure corresponding to this case). The pivot and ghost vertices are given by $w(0,1)=\left(a_{1}, b_{1}\right)$ and $v(n, 1)=\left(a_{n}+p_{n}, b_{n}+q_{n}\right)$, respectively.

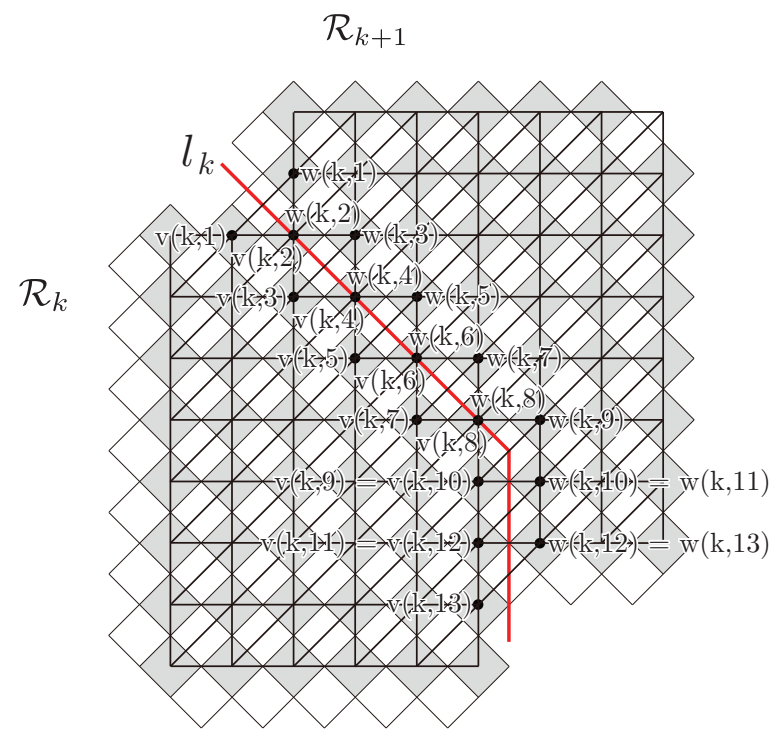

Figure 11: Two families of vertices near $l_{k}$

In the next theorem, $\left[\rho_{v(k, j)-w(k-1, i)}\right]$ is an $M_{k-1} \times M_{k}$ matrix whose entries $\rho_{v(k, j)-w(k-1, i)}$ indicate the number of Delannoy paths from $w(k-1, i)$ to $v(k, j)$ in $\Gamma_{\left(p_{k}, q_{k}\right)}\left(a_{k}, b_{k}\right)$. For example, when $1 \leqslant i \leqslant m_{k-1}$ and $1 \leqslant j \leqslant m_{k}$,

$$
\rho_{v(k, j)-w(k-1, i)}=\rho_{\left(a_{k+1}-a_{k}-\left\lfloor\frac{i+1}{2}\right\rfloor+\left\lfloor\frac{j}{2}\right\rfloor, b_{k}-b_{k-1}+q_{k}-q_{k-1}+\left\lfloor\frac{i}{2}\right\rfloor-\left\lfloor\frac{j+1}{2}\right\rfloor\right)} \cdot
$$

Theorem 7. There is a bijection between domino tilings for a horizontal chain

$$
\bigcup_{k=1}^{n} \mathcal{R}_{\left(p_{k}, q_{k}\right)}\left(a_{k}, b_{k}\right)
$$

and Delannoy paths from $\left(a_{1}, b_{1}\right)$ to $\left(a_{n}+p_{n}, b_{n}+q_{n}\right)$ in its dual lattice graph $\Gamma_{h}$. Furthermore, the number of domino tilings for this chain is equal to the sole entry of the $1 \times 1$ matrix obtained by multiplying $n$ matrices

$$
\prod_{k=1}^{n}\left[\rho_{v(k, j)-w(k-1, i)}\right]_{1 \leqslant i \leqslant M_{k-1}, 1 \leqslant j \leqslant M_{k}} .
$$


Proof. As before, we define a map $\Phi_{h}$ from the set of domino tilings for this horizontal chain to the set of Delannoy paths from $\left(a_{1}, b_{1}\right)$ to $\left(a_{n}+p_{n}, b_{n}+q_{n}\right)$ in $\Gamma_{h}$.

Given a domino tiling $T$, we perform the path extension process starting from the pivot vertex $\left(a_{1}, b_{1}\right)$. Then we get the uniquely defined Delannoy path $P$ reaching the ghost vertex $\left(a_{n}+p_{n}, b_{n}+q_{n}\right)$ by Claim 2 . Note that $P$ goes through an extra edge $e_{i}$ (or $e_{i}^{\prime}$ ) whenever the black square centered at $\left(a_{i+1}-1, b_{i}+q_{i}\right)\left(\right.$ or $\left(a_{i}+p_{i}, b_{i+1}-1\right)$, respectively) is covered by an ne-domino in $T$. As in the proof of Theorem 1 , the dominoes in $T$ away from $P$ are all sw-dominoes. Therefore $\Phi_{h}(T)$ is a Delannoy path in $\Gamma_{h}$.

The injectivity of $\Phi_{h}$ follows immediately from the Delannoy path replacement. For the surjectivity, we construct a domino tiling for the horizontal chain from a given Delannoy path $P$ from $\left(a_{1}, b_{1}\right)$ to $\left(a_{n}+p_{n}, b_{n}+q_{n}\right)$ in $\Gamma_{h}$ by reversing the Delannoy path replacement. As before, let $\mathcal{R}_{P}$ be the union of the D-dominoes that cover $P$ and $\mathcal{R}_{P}^{c}$ the complementary region of $\mathcal{R}_{P}$. By Lemma 4 , the union $\bigcup_{k=1}^{n} \mathcal{R}_{\left(p_{k}, q_{k}\right)}\left(a_{k}, b_{k}\right)$ has the same number of black and white squares, and so does $\mathcal{R}_{P}^{c}$. For any white square in $\mathcal{R}_{P}^{c}$, the black square which is ne-adjacent to the white square is also contained in $\mathcal{R}_{P}^{c}$. This implies that $\mathcal{R}_{P}^{c}$ can be tiled only by sw-dominoes, which guarantees that there is a domino tiling of $\bigcup_{k=1}^{n} \mathcal{R}_{\left(p_{k}, q_{k}\right)}\left(a_{k}, b_{k}\right)$ whose image under $\Phi_{h}$ is the Delannoy path $P$.

From now on, we count the number of Delannoy paths from $\left(a_{1}, b_{1}\right)$ to $\left(a_{n}+p_{n}, b_{n}+\right.$ $\left.q_{n}\right)$ in $\Gamma_{h}$. First we consider $n+1$ families of points in $\Gamma_{h}, C_{0}=\left\{c(0,1)=\left(a_{1}, b_{1}\right)\right\}$, $C_{k}=\left\{c(k, 1), c(k, 2), \ldots, c\left(k, M_{k}\right)\right\}$ where $c(k, i)=\frac{v(k, i)+w(k, i)}{2}$ for $k=1,2, \ldots, n-1$, and $C_{n}=\left\{c(n, 1)=\left(a_{n}+p_{n}, b_{n}+q_{n}\right)\right\}$. Note that $c(k, 1)$ and $c\left(k, M_{k}\right)$ are the center points of $e_{k}$ and $e_{k}^{\prime}$, respectively.

The crucial point is that any Delannoy path $P$ from $\left(a_{1}, b_{1}\right)$ to $\left(a_{n}+p_{n}, b_{n}+q_{n}\right)$ in $\Gamma_{h}$ always passes through $l_{k}$ at exactly one point, say $c\left(k, i_{k}\right)$, of $C_{k}$ for each $k=1,2, \ldots, n-1$. Let $c\left(0, i_{0}\right)=c(0,1)$ and $c\left(n, i_{n}\right)=c(n, 1)$. The points $c\left(k, i_{k}\right)$ break $P$ into $n$ consecutive subpaths $P_{k}, k=1,2, \ldots, n$, starting at $c\left(k-1, i_{k-1}\right)$ and ending at $c\left(k, i_{k}\right)$. See Figure 12 . The number of such subpaths $P_{k}$ joining $c\left(k-1, i_{k-1}\right)$ and $c\left(k, i_{k}\right)$ equals the number of Delannoy paths from $w\left(k-1, i_{k-1}\right)$ to $v\left(k, i_{k}\right)$ in $\Gamma_{\left(p_{k}, q_{k}\right)}\left(a_{k}, b_{k}\right)$. This number is exactly $\rho_{v\left(k, i_{k}\right)-w\left(k-1, i_{k-1}\right)}$ by Theorem 1. We write $\rho_{i_{k-1} i_{k}}^{k}$ for $\rho_{v\left(k, i_{k}\right)-w\left(k-1, i_{k-1}\right)}$ in short.

Therefore the total number of Delannoy paths from $w(0,1)=\left(a_{1}, b_{1}\right)$ to $v(n, 1)=$ $\left(a_{n}+p_{n}, b_{n}+q_{n}\right)$ in $\Gamma_{h}$ is

$$
\sum_{i_{1}=1}^{M_{1}} \sum_{i_{2}=1}^{M_{2}} \cdots \sum_{i_{n-1}=1}^{M_{n-1}} \rho_{1 i_{1}}^{1} \rho_{i_{1} i_{2}}^{2} \cdots \rho_{i_{n-2} i_{n-1}}^{n-1} \rho_{i_{n-1} 1}^{n},
$$

which equals the sole entry of the $1 \times 1$ matrix obtained by the matrix multiplication

$$
\prod_{k=1}^{n}\left[\rho_{i j}^{k}\right]_{1 \leqslant i \leqslant M_{k-1}, 1 \leqslant j \leqslant M_{k}} .
$$

This completes the proof. 


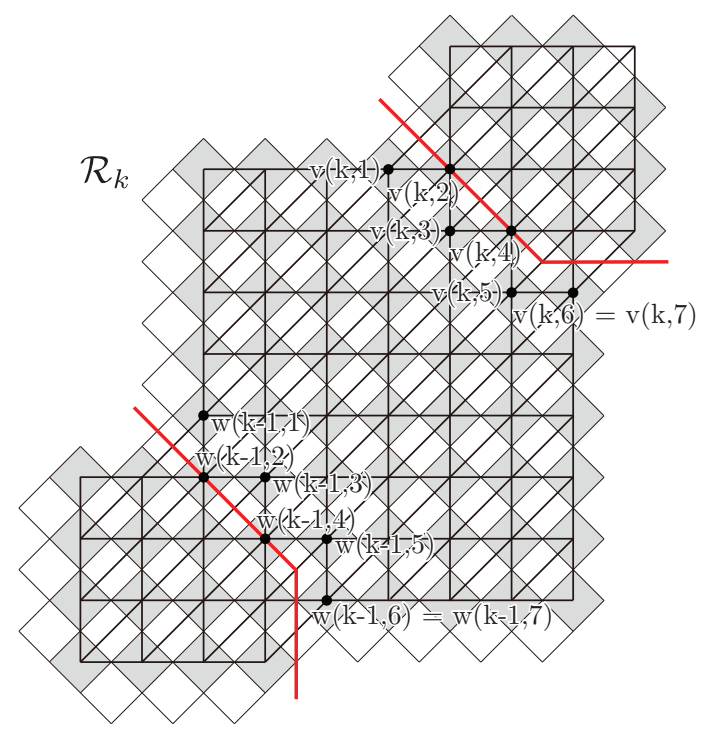

Figure 12: Subpaths $P_{k}$

Corollary 8. The number of domino tilings for a simple horizontal chain

$$
\bigcup_{k=1}^{n} \mathcal{R}_{\left(p_{k}, q_{k}\right)}\left(a_{k}, b_{k}\right)
$$

equals the sole entry of the $1 \times 1$ matrix obtained by multiplying $n$ matrices

$$
A_{1} A_{2} \cdots A_{n}
$$

where

$$
\begin{gathered}
A_{1}=\left[\begin{array}{lll}
\rho_{\left(p_{1}-1, q_{1}\right)} & \rho_{\left(p_{1}, q_{1}\right)} & \rho_{\left(p_{1}, q_{1}-1\right)}
\end{array}\right], \\
A_{n}=\left[\begin{array}{lll}
\rho_{\left(p_{n}, q_{n}-1\right)} & \rho_{\left(p_{n}, q_{n}\right)} & \rho_{\left(p_{n}-1, q_{n}\right)}
\end{array}\right]^{T},
\end{gathered}
$$

and

$$
A_{k}=\left[\begin{array}{ccc}
\rho_{\left(p_{k}-1, q_{k}-1\right)} & \rho_{\left(p_{k}, q_{k}-1\right)} & \rho_{\left(p_{k}, q_{k}-2\right)} \\
\rho_{\left(p_{k}-1, q_{k}\right)} & \rho_{\left(p_{k}, q_{k}\right)} & \rho_{\left(p_{k}, q_{k}-1\right)} \\
\rho_{\left(p_{k}-2, q_{k}\right)} & \rho_{\left(p_{k}-1, q_{k}\right)} & \rho_{\left(p_{k}-1, q_{k}-1\right)}
\end{array}\right]
$$

for $k=2, \ldots, n-1$.

Proof. For each $k=1, \ldots, n-1$, since $a_{k+1}=a_{k}+p_{k}$ and $b_{k+1}=b_{k}+q_{k}$, we have $m_{k}=M_{k}=3$. Furthermore, $v(k, 1)=\left(a_{k+1}-1, b_{k}+q_{k}\right), w(k, 1)=\left(a_{k+1}, b_{k}+q_{k}+1\right)$, $v(k, 2)=w(k, 2)=\left(a_{k+1}, b_{k}+q_{k}\right), v(k, 3)=\left(a_{k+1}+1, b_{k}+q_{k}-1\right)$ and $w(k, 3)=\left(a_{k+1}+\right.$ $\left.1, b_{k}+q_{k}\right)$. Recall that $M_{0}=M_{n}=1, w(0,1)=\left(a_{1}, b_{1}\right)$ and $v(n, 1)=\left(a_{n}+p_{n}, b_{n}+q_{n}\right)$.

A simple calculation shows that for $k=1, \ldots, n$

$$
\left[\rho_{v(k, j)-w(k-1, i)}\right]_{1 \leqslant i \leqslant M_{k-1}, 1 \leqslant j \leqslant M_{k}}=A_{k},
$$

where $A_{k}$ is as stated in this corollary. 


\section{References}

[1] F. Bosio and M. Leeuwen. A bijective proving the Aztec diamond theorem by combing lattice paths. Electron. J. Comb., 20(4):\#P24, 2013.

[2] R. Brualdi and S. Kirkland. Aztec diamonds and digraphs, and Hankel determinants of Schröder numbers. J. Comb. Theory Ser. B, 94:334-351, 2005.

[3] S. Eu and T. Fu. A simple proof of the Aztec diamond theorem. Electron. J. Comb., 12:\#R18, 2005.

[4] N. Elkies, G. Kuperberg, M. Larsen, and J. Propp. Alternating-sign matrices and domino tilings (parts I and II). J. Algebr. Comb., 1:111-132 and 219-234, 1992.

[5] M. Fendler and D. Grieser. A new simple proof of the Aztec diamond theorem. Graphs Comb., 32:1389-1395, 2016.

[6] I. Gessel and G. Viennot. Binomial determinants, paths, and hook length formulae. Adv. Math., 58:300-321, 1985.

[7] I. Gessel and G. Viennot. Determinants, Paths, and Plane Partitions. a Brandeis University report, available from http://contscience.xavierviennot.org, 1989.

[8] D. Grensing, I. Carlsen, and H. Zapp. Some exact results for the dimer problem on plane lattices with non-standard boundaries. Philosophical Magazine A, 41:777-781, 1980.

[9] W. Jockusch, J. Propp, and P. Shor. Random domino tilings and the Arctic circle theorem. arXiv:math/9801068, 1998.

[10] B. Lindström. On the vector representations of induced matroids. Bull. London Math. Soc., 5:85-90, 1973.

[11] R. Merrifield and H. Simmons. Enumeration of structure-sensitive graphical subsets: Theory. Proc. Natl. Acad. Sci. USA, 78:692-695, 1981.

[12] R. Merrifield and H. Simmons. Enumeration of structure-sensitive graphical subsets: Calculations. Proc. Natl. Acad. Sci. USA, 78:1329-1332, 1981.

[13] J. Propp. Enumeration of matchings: problems and progress. New Perspectives in Geometric Combinatorics, MSRI Publications, 38:255-290, 1999.

[14] H. Sachs and H. Zernitz. Remark on the dimer problem. Discrete Appl. Math., 51:171-179, 1994. 